\title{
Shortness of breath caused by a tracheal web
}

\author{
Nagham Jafar MD, Ebtesam Islam MD, PhD, Raed Alalawi MD
}

\begin{abstract}
Tracheal webs are rare anomalies that can be either congenital or acquired due to prolonged or traumatic endotracheal intubation. Patients with tracheal webs present with shortness of breath especially during exertion, stridor, and recurrent chest infections. They are often misdiagnosed and treated as bronchial asthma with only minimal relief of symptoms with bronchodilators. This condition can also present as a cause of difficult endotracheal intubations. CT scans of the chest can be used to screen for airway anomalies; bronchoscopy is both diagnostic and therapeutic. Here we present a 34-year-old woman with a tracheal web who had been on treatment for refractory asthma for many years with only partial relief of her symptoms.
\end{abstract}

Key words: refractory asthma, tracheal web, bronchoscopy

\section{INTRODUCTION}

Congenital tracheal webs are very rare and often misdiagnosed. These patients usually present with wheezing, stridor, recurrent chest infections, and even respiratory failure. ${ }^{1}$ Here we present a 34 -yearold woman who had been on treatment for refractory asthma for many years with only partial relief of her symptoms.

\section{Case Presenttion}

A 34-year-old woman with a history of periodic wheezing and dyspnea for the past 10 years was diagnosed with asthma and treated with corticosteroid inhalers and bronchodilators with some improvement in her symptoms. Her symptoms became progressively worse after her last pregnancy at age 32 , and

Corresponding author: Raed Alalawi MD Contact Information: raed.alalawi@ttuhsc.edu DOI: 10.12746/swrccc2015.0309.122 optimizing her medications didn't relieve her symptoms. The patient quit smoking 10 years ago. She had a past history of an appendectomy in 1993 for which she was intubated without difficulty. Her family history was relevant as her mother experienced the same symptoms. On physical examination, the patient had audible wheezing and stridor. Her lung bases were clear to auscultation.

Her chest x-ray was unremarkable. Pulmonary function tests showed a normal FEV1/FVC ratio and DICO. The flow-volume loops showed some flattening of the inspiratory and expiratory limbs, suggestive of upper airway obstruction. A CT scan of the neck and chest revealed a thin air filled cystic structure at the C5 level consistent with a tracheal web $2.5 \mathrm{~cm}$ below the hyoid bone (Figure 1). Bronchoscopy confirmed the tracheal web (Figure 2), and it was successfully removed using cautery, cryotherapy, and balloon dilatation with resolution of the patient's symptoms (Figure 3). On follow up in the clinic, the patient's dyspnea had resolved; she had no limitations in her daily activity or with exercise. 


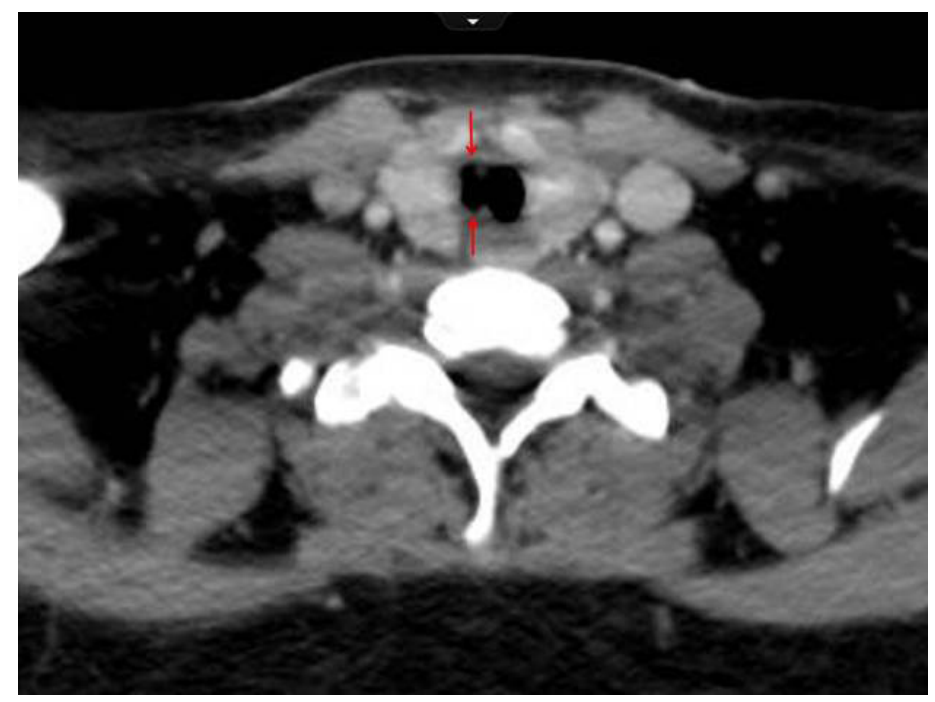

Figure 1. CT scan of the chest. Arrows indicate the attachment of the tracheal web to the tracheal wall.

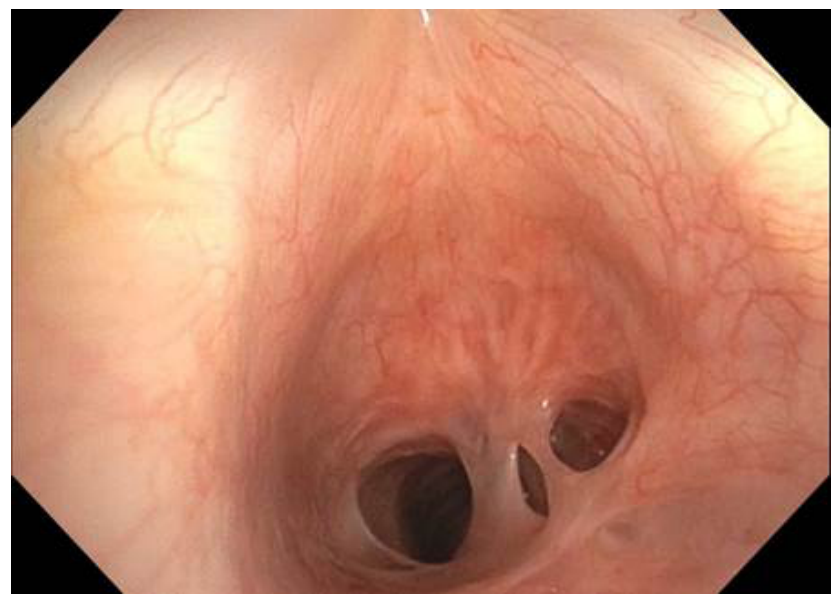

Figure 2. Tracheal web

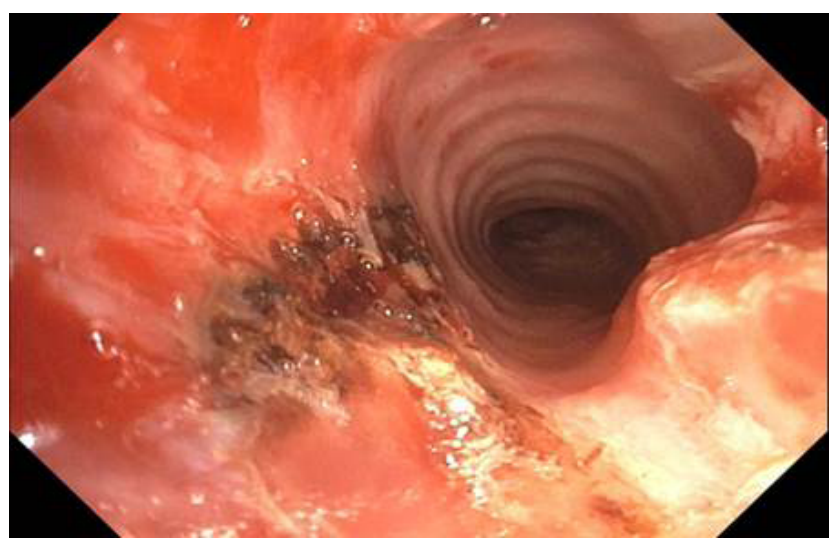

Figure 3. Removal of tracheal web using cautery, balloon dilatation, and cryotherapy

\section{DIscussion}

Congenital tracheal malformations can be either an intrinsic disorder in the trachea or an extrinsic disorder compressing the airway. Congenital tracheal webs are rare with an incidence of 1 in 10,000 births; they are usually undiagnosed in infancy and often misdiagnosed later. ${ }^{2}$ A tracheal web is formed by a thin layer of membranous tissue containing small holes that cause the tracheal lumen to narrow leading to partial airway obstruction. Affected patients usually complain of wheezing, dyspnea, stridor, recurrent respiratory infections, and sometimes respiratory failure. ${ }^{1}$ This anomaly usually presents in the pediatric population.

In adults tracheal webs are usually due to prolonged or traumatic endotracheal intubation. ${ }^{2}$ Patients are often misdiagnosed as having refractory asthma or chronic obstructive lung disease due to the wheezing and the recurrent chest infections. These patients can also be asymptomatic and diagnosed accidentally after difficult endotracheal intubations for surgical procedures. ${ }^{3,4}$ The position of the tracheal web also influences the patient's symptoms. Higher level (extrathoracic/cervical) webs cause symptoms in the inspiratory phase, and lower level (intrathoracic) webs cause symptoms in the expiratory phase.

Tracheal stenosis is seldom detected by conventional chest $x$-ray, but this condition is easily detected on CT scans which can also help determine the anatomy and the underlying cause of tracheal stenosis. ${ }^{5}$ Pulmonary function tests with a high FEV1 to $\mathrm{PF}$ ratio and a poor response to bronchodilators suggest the possibility of obstructive tracheal lesions and indicate the need for additional studies. ${ }^{6}$ Empey has suggested that a FEV1/PF ratio $\geq 10$ is useful for screening for upper airway obstruction and that a plateau pattern in the inspiratory and expiatory limbs of flow volume loops might indicate central airway obstruction. ${ }^{7}$ Quint showed that a CT scan with multiplanar reconstruction is $90 \%$ accurate for the diagnosis of the anomaly and also shows the morphology and extent of tracheal stenosis. ${ }^{8}$ A three dimensional CT scan was also used for diagnosis in a case of tracheal web reported by Yang. ${ }^{9}$ Thus CT scans should 
be employed as a screening tool in case of tracheal deformities and stenosis. Bronchoscopy is the gold standard for diagnosing tracheal webs.

The youngest patient reported in the literature is a 74-day-old girl. ${ }^{1}$ Here we report a 34-year-old woman who was misdiagnosed and treated for refractory bronchial asthma for many years with progressive worsening of her symptoms after pregnancy. A CT scan showed the possibility of a tracheal web which was confirmed and treated by bronchoscopy. The patient had a complete resolution of her symptoms and discontinued her medications.

\section{KEYPOINTS}

1. The presence of respiratory symptoms like wheezing very early in life or during adulthood, especially in refractory cases, should raise suspicion for tracheal stenosis.

2. A high FEV1/PF $(\geq 10)$ ratio should point toward the need for further work up when evaluating a patient with refractory asthma.

3. CT scans can be used for screening for tracheal webs and other tracheal anomalies and can provide information about the morphology and extent of the lesion.

4. Bronchoscopy is the gold standard in diagnosing tracheal webs and can provide immediate therapy.

Author Affiliation: All authors work in the Department of Internal Medicine at Texas Tech University Health Sciences Center in Lubbock, TX. Nagham Jafar is a research associate, Ebtesam Islam is a fellow in Pulmonary and Critical Care Medicine, and Raed Alalawi is a faculty member in the Division of Pulmonary and Critical Care Medicine.

Received: $12 / 22 / 2014$

Accepted: 01/03/2015

Reviewers: Kenneth Nugent MD

Published electronically: 01/15/2015

Conflict of Interest Disclosures: none

\section{REFERENCES}

1. Yin Y, Zhang L. Successful diagnosis and treatment of congenital tracheal web using a fiberoptic bronchoscope. Pediatr Pulmonol 2010; 45(9):945-947.

2. Kokkonouzis I, Mermigkis C, Psathakis K, Tsintiris K. Postintubation tracheal web. J Broncho 2005; 12(4):271-272.

3. Al Badaai Y, Nguyen LH. Difficult intubation due to undiagnosed congenital tracheal web. Int $J$ Ped Otorhinolaryngology 2008; 72(9):1437-1439.

4. Egawa A, Hirabayashi Y, Seo N. Case of unexpected difficult intubation caused by asymptomatic congenital laryngeal web. Masui 2011; 60(10):1211-3.

5. Legasto AC, Haller JO, Giusti RJ. Tracheal web. Pediatr Radiol 2004; 34(3): 256-258.

6. Linna O, Hyrynkangas $K$, Lanning P, Nieminen P. Central airways stenosis in school-aged children: differential diagnosis from asthma. Acta Pediatr 2002; 91(4):399-402.

7. Empey DW. Assessment of upper airways obstruction. Br Med $J$ 1972; 3: 503-5.

8. Quint LE, Whyte RI, Kazerooni EA, Martinez FJ, Cascade PN, Lynch JP III, Orringer MB, Brunsting LA III, Deeb GM. Stenosis of the central airways: evaluation by using helical CT with multiplanar reconstructions. Radiology 1995; 194: 871-7.

9. Yang C, Fan CY, Xu MS. The clinical evaluation of CT virtual endoscopy combined with bronchial three-dimensional reconstruction. Jiangsu Med J 2001; 27: 519-520. 